\title{
¿Tiene este paciente una disección aguda de aorta torácica?
}

Does this patient have an acute thoracic aortic dissection? Klompas M. JAMA 2002;287(17):2262-2272.

\section{Objetivo}

Revisar la utilidad del interrogatorio, examen físico y radiografía de tórax para el diagnóstico de disección aguda de aorta toráci$\mathrm{ca}(\mathrm{DAAT})$

\section{Diseño}

Revisión de la literatura publicada en inglés entre 1966 y 2000 y las citas de esos artículos, utilizando el MEDLINE.

\section{Lugar}

Departamento de Medicina, Brigham and Women's Hospital, Boston, Mass.

\section{Selección de los artículos}

La búsqueda original detectó 274 artículos posibles.Sólo fueron incluidos los artículos originales, con descripción de 18 pacientes ó más, con diagnóstico confirmado de DAAT. Veintiún artículos cumplieron con los criterios de inclusión y de estos sólo 10 fueron originalmente diseñados con el objetivo de describir la forma de presentación de la DAAT.Un sólo artículo fue un estudio prospectivo.

\section{Medición de Resultados}

Se obtuvo la sensibilidad, el coeficiente de probabilidad positivo (CPP) y negativo (CPN) * de los diferentes hallazgos. Sólo 4 estudios incluyeron datos de especificidad que permitieron construir las tasas de probabilidad.

Resultados Principales
\begin{tabular}{|l|l|l|l|l|l|l|l}
\hline Hallazgo & $\begin{array}{l}\text { Dolor } \\
\text { severo }\end{array}$ & $\begin{array}{l}\text { Inicio } \\
\text { brusco }\end{array}$ & $\begin{array}{l}\text { Ausencia } \\
\text { de dolor } \\
\text { al inicio }\end{array}$ & $\begin{array}{l}\text { Déficit de } \\
\text { un pulso ó } \\
\text { diferencia } \\
\text { de TA }\end{array}$ & $\begin{array}{l}\text { Foco } \\
\text { neurológico }\end{array}$ & $\begin{array}{l}\text { RxTx } \\
\text { Anormal }\end{array}$ & $\begin{array}{l}\text { RxTx } \\
\text { Normal }\end{array}$ \\
\hline Sensibilidad & $90 \%$ & $84 \%$ & $10 \%$ & $31 \%$ & $17 \%$ & $90 \%$ & $10 \%$ \\
\hline CPN & & & 0,3 & & & & 0,3 \\
$(0,2-0,5)$ & & & \\
\hline CPP & & 1,6 & & $5,20,5)$ \\
\hline$(1-2,4)$ & & 5,7 & 6,6 & & \\
\hline
\end{tabular}

El antecedente de hipertensión y la presencia de soplo diastólico no resultaron de utilidad para el diagnóstico.

El antecedente de enfermedad de Marfán fue poco frecuente, pero su presencia incrementó la probabilidad de DAAT.

\section{Conclusiones}

1) La ausencia de dolor intenso y de inicio brusco como forma de presentación aleja la posibilidad del diagnóstico de DAAT.

2) Menos de un tercio de los pacientes presentan déficit de un pulso ó diferencia en la presión arterial entre los dos brazos; sin embargo cuando estos hallazgos están presentes, aumentan significativamente la probabilidad de DAAT.

3) La presencia de una radiografía de tórax normal aleja considerablemente el diagnóstico.

\section{Comentario}

El dolor torácico es uno de los motivos de consulta más frecuentes en el departamento de emergencias.Si bien la mayoría de estos casos corresponden a patologías benignas y de buen pronóstico, uno de los diagnósticos diferenciales que debe ser reconocido es la DAAT.

Si bien las características clínico-radiológicas de la DAAT están extensamente descriptas, no existen datos de ensayos prospectivos que permitan utilizar los hallazgos del interrogatorio, examen físico y radiografía de tórax para certificar ó excluir el diagnóstico con certeza.

Aunque existen varios métodos complementarios que pueden confirmar el diagnóstico (aortografía, TAC y RMN de tórax y ecocardiograma transesofágico) no siempre están disponibles, algunos son invasivos ó requieren contraste y en todos los casos son costosos. De manera que la utilización indiscriminada en todos los pacientes con dolor torácico no es costo-efectiva. Para racionalizar la evaluación adquiere relevancia conocer la sensibilidad y coeficientes de probabilidad de los diferentes hallazgos clínicos en la DAAT.

Este estudio concluyó que la utilidad de cada uno de los hallazgos típicos (dolor, ausencia de pulso ó diferencia en la presión arterial y radiografía de tórax anormal) es escasa si se consideran aisladamente, pero que la combinación de los mismos aumenta mucho la probabilidad de DAAT (CPP 6,6).

Por otro lado, aunque la ausencia combinada de los tres aleja mucho la probabilidad del diagnóstico (CPN de 0,07;0,03-0,7), debido a que se trata de una patología con alta mortalidad, no es posible recomendar suspender la evaluación en estos pacientes, pues hasta el $4 \%$ de los mismos tendrán una DAAT como diagnóstico final.

Conclusión del comentador: En resumen, la DAAT es una entidad infrecuente pero de diagnóstico difícil y alta mortalidad.Sus manifestaciones clínicas son inespecíficas y los síntomas clásicos frecuentemente están ausentes.

La combinación de los hallazgos típicos obliga a certificar el diagnóstico con algún método complementario, pero la ausencia combinada de los mismos no tiene suficiente poder como para descartar por completo esta entidad.

Para su diagnóstico precoz se requiere siempre un alto índice de sospecha.

\section{Dr. Fernando Vázquez [ Servicio de Clínica Médica.Hospital Italiano de Buenos Aires ]}

Referencias

1.Von Kodolitsch Y, Schwartz AG, Nienaber CA.Clinical prediction of acute aortic dissection.Arch Intern Med. 2000;160:2977-2982.

2. Hagan P, Nienaber CA, Isselbacher EM et al.The internacional registry of acute aortic dissection (IRAD). New insights into an old disease. 2000;283:897-903. 